Supporting Information

\title{
Synthesis of High Purity Few-Walled Carbon Nanotubes from Ethanol/Methanol Mixture
}

\author{
Hang Qi, Cheng Qian, Jie Liu*
}

Department of Chemistry, Duke University, Durham NC 27708

\author{
J.Liu@duke.edu
}



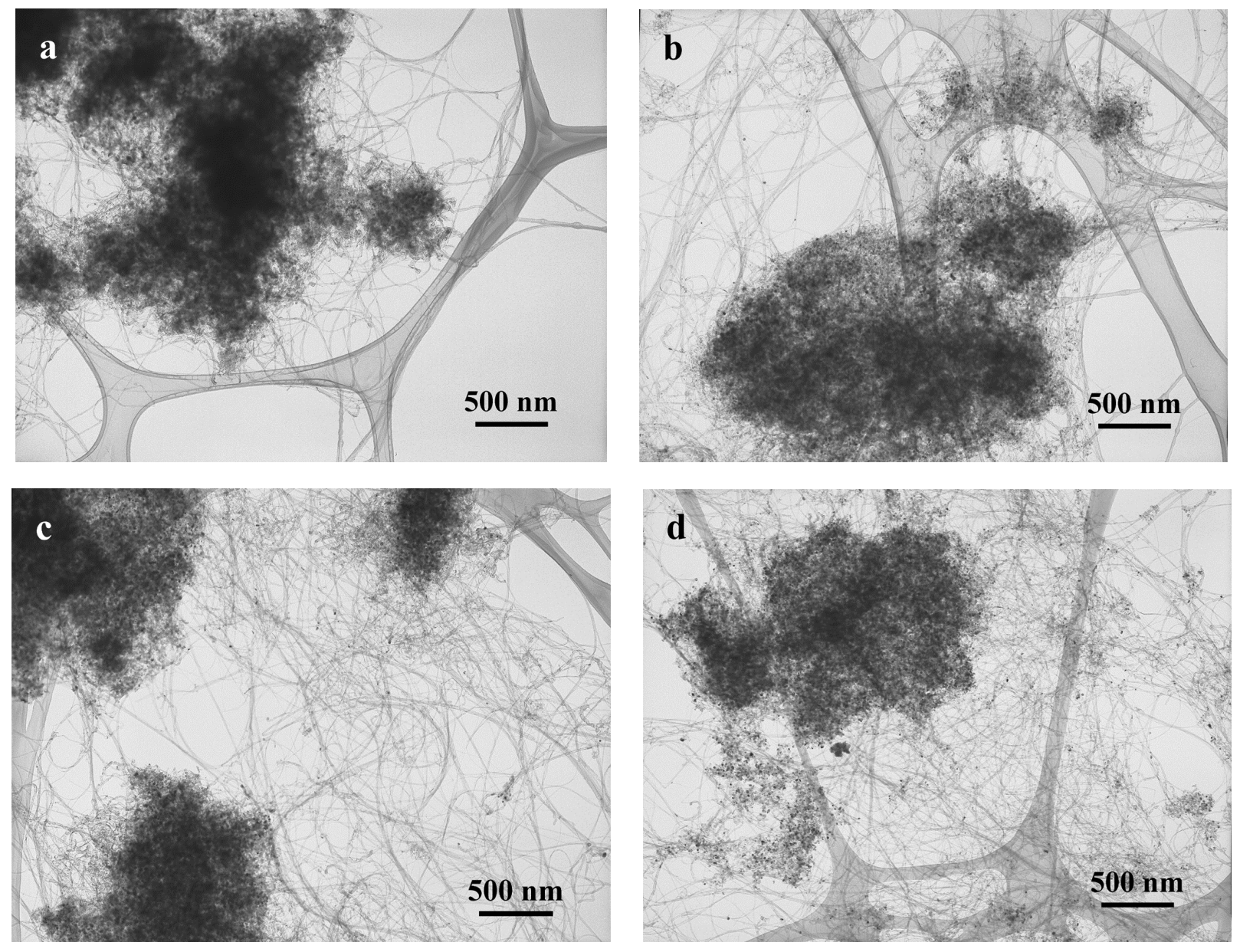

Figure S1, TEM images of raw material which are treated with $6 \mathrm{M} \mathrm{HCl}$. The raw material was prepared by using carbon source with different ethanol concentration, a) 100 vol\% ethanol, b) 80 vol\% ethanol, c) 50 vol\% ethanol and d) 20 vol\% ethanol. 

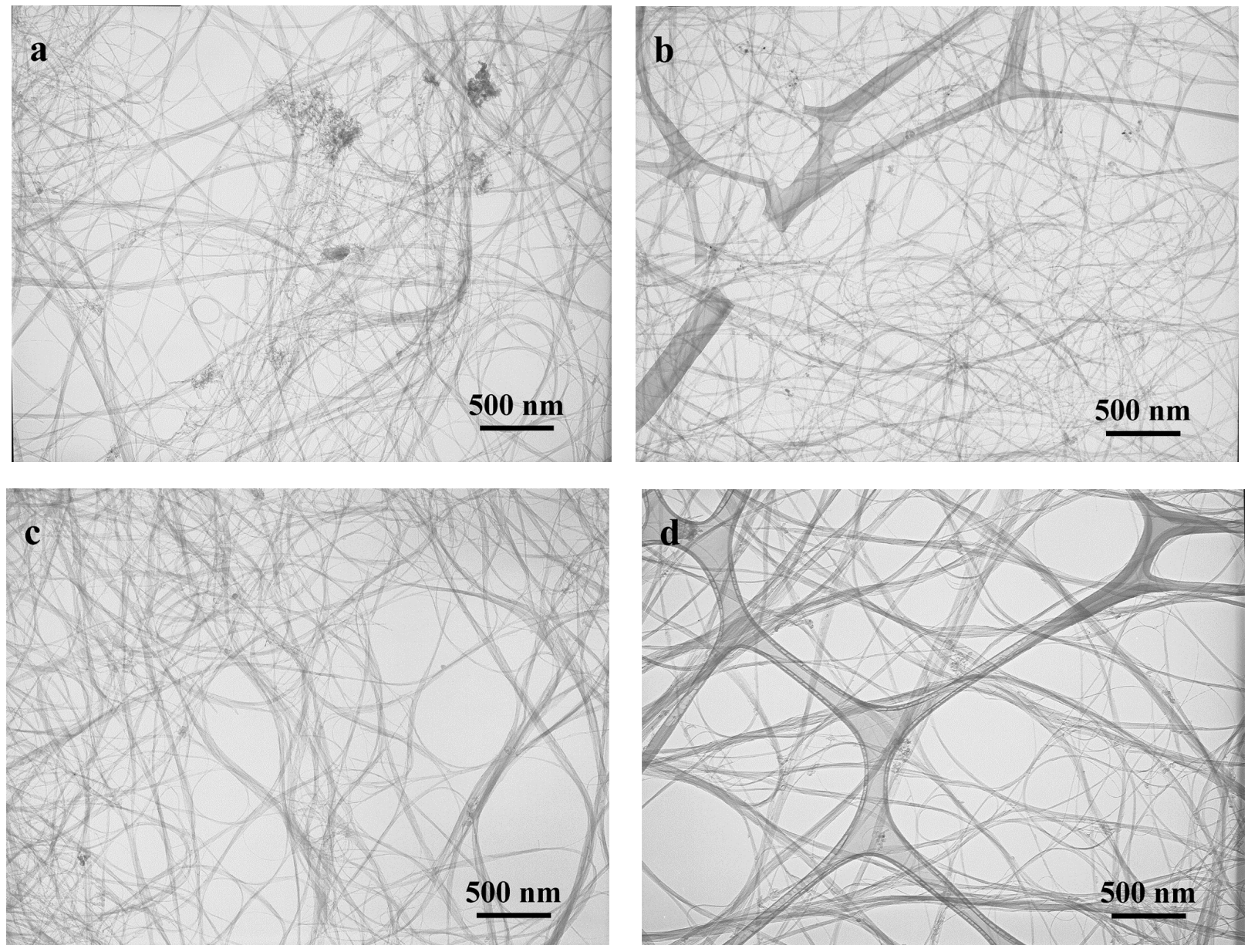

Figure S2, TEM images of purified samples from raw material using carbon source with different ethanol concentration, a) $100 \mathrm{vol} \%$ ethanol, b) $80 \mathrm{vol} \%$ ethanol, c) $50 \mathrm{vol} \%$ ethanol and d) $20 \mathrm{vol} \%$ ethanol. 

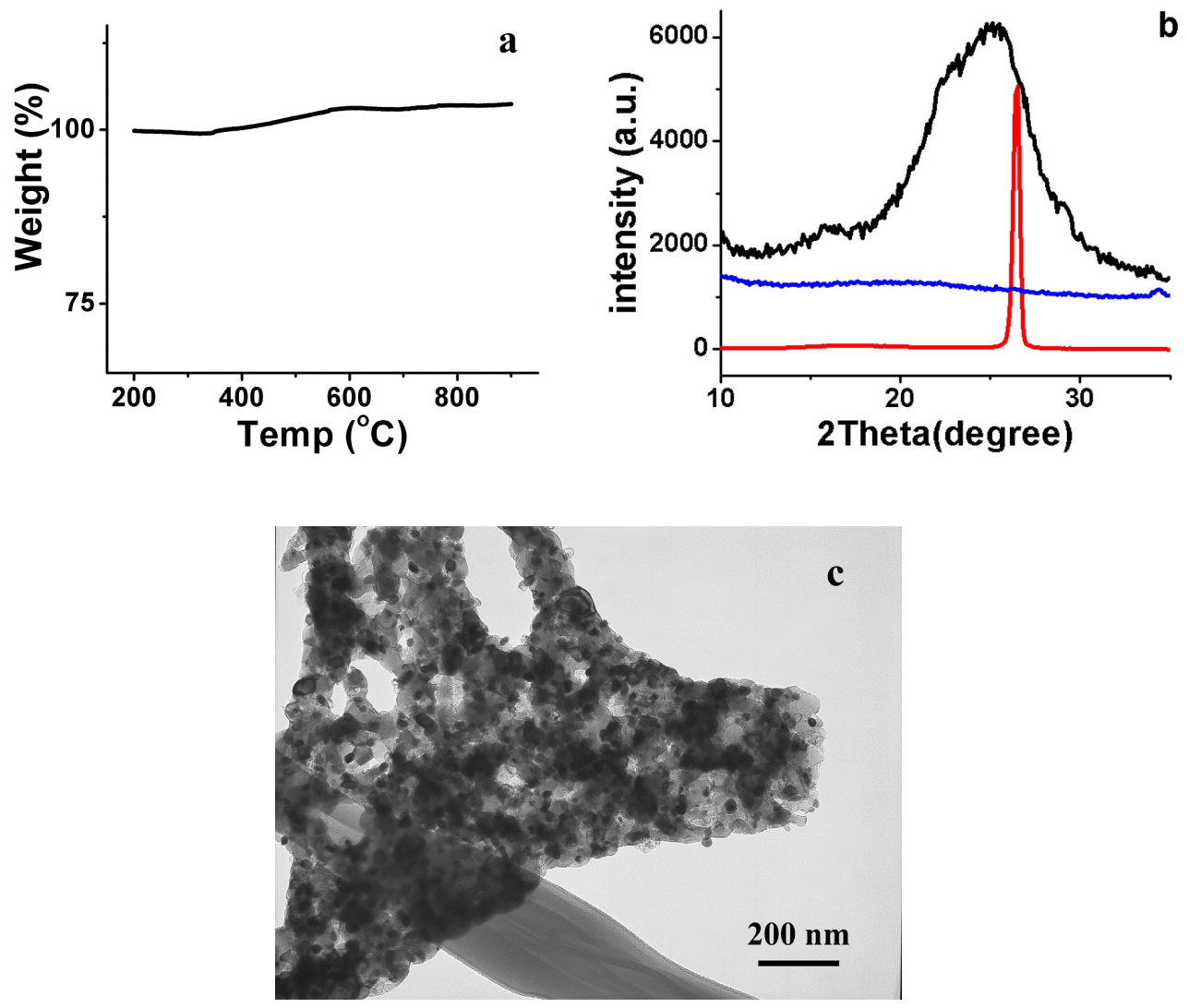

Figure S3, TGA weight loss plot (a), XRD (b) and TEM image (c) of methanol sample. The TGA plot (a) shows an increase instead of decrease suggest there are almost no carbonaceous species and the increase is due to oxidization of metal in the catalyst. Blue, black and red plots in b are XRD plot of methanol sample, purified FWNTs and graphite, respectively; the peak around $26.5^{\circ}(\mathrm{red})$ is the character of carbon with graphite sheet, broadening and down shift of this peak (black) is due to the bend and small scale of graphite sheets in FWNTs. Absence of peak at $26.5^{\circ}$ in blue plot suggests there is almost no carbon in methanol sample. 